\title{
O Que Está Sendo Aprendido a Respeito da Dor na Uel?
}

\section{What is Being Learned About Pain in the State University of Londrina?}

\author{
Talita Woitas Sereza ${ }^{1}$; Mara Solange Gomes Dellaroza ${ }^{2}$
}

Resumo

\begin{abstract}
A dor acomete todas as pessoas em algum momento da vida e por vezes acarreta sérias complicações, sendo assim, a principal causa por procura de Serviços de Saúde. O objetivo deste estudo foi verificar os conhecimentos adquiridos acerca da dor pelos alunos da área de saúde da UEL. Para tal, foi aplicado questionário previamente validado com perguntas objetivas aos alunos do último ano de graduação sobre: aspectos gerais, fisiopatologia, avaliação e terapêuticas para dor. Dos acadêmicos avaliados, 81,5\% referem que o tema dor foi discutido em sua formação acadêmica, 53,4\% dos entrevistados consideraram a dor como $5^{\circ}$ sinal vital. Além disso, $50,8 \%$ dos alunos acreditam que o paciente tem sua dor controlada. Em relação a terapêutica, $36 \%$ afirmam que a morfina deve ser utilizada somente como última alternativa no tratamento da dor e $10 \%$ não souberam informar se as técnicas de distração podem promover o alívio da dor. Pela análise percebe-se que os acadêmicos apresentam adequados conceitos sobre a fisiopatologia e avaliação da dor, porém com conceitos errôneos sobre terapêuticas de dor e com mitos sobre o uso de opiódes. O tema dor é essencial para estes acadêmicos, já que estarão cuidando da população. Assim, é urgente rever como o tema é abordado nos cursos de graduação em saúde.

Palavras-chave: Dor. Profissionais de saúde. Educação.
\end{abstract}

\begin{abstract}
The pain attacks everybody sometime in a lifetime and it sometimes causes serious complications, being the main reason for searching Health (Care) Services. The purpose of this study was to verify the knowledge acquired about pain by the students from the health area in the State University of Londrina. In order to do so, it was applied a questionnaire validated with objective questions to the students of the last year of graduation about: general aspects, physiopathology, evaluation and therapies for pain relief. Among the students who were evaluated, $81,5 \%$ mentioned that this subject was covered in their academic formation; $53,4 \%$ considered pain as the $5^{\text {th }}$ vital sign. Moreover, $50,8 \%$ believe that the patient has adequate control over pain. In terms of therapy, $36 \%$ claim that the morphine should be used as a last resource in the treatment of pain and $10 \%$ could not inform if the distraction techniques can relieve pain. By the analysis it could be noticed that the students have adequate concepts about the physiopathology and evaluation of pain, but wrong concepts about therapies for the pain and with myths about the use of opioids. This theme is extremely important so it is essential to check how it is taught in health graduation courses.
\end{abstract}

Key words: Pain. Professionals of health. Education.

\footnotetext{
${ }^{1}$ Graduanda do $4^{\circ}$ ano do Curso de Enfermagem da Universidade Estadual de Londrina. ${ }^{2}$ Docente do Departamento de engermagem da Universidade Estadual de Londrina
} 


\section{Introdução}

Quem nunca sentiu dor? Muito se tem discutido sobre dor nos dias de hoje e muitos avanços neste sentido já foram alcançados. Está cada vez mais evidente a importância deste tema que atinge as pessoas desde a antigüidade. Mas será que as pessoas em geral estão preparadas para lidar com a dor? E os profissionais de saúde, estão eles capacitados para isso?

A dor é a principal causa que leva indivíduos a procurar os serviços de saúde. A dor não escolhe idade, sexo, raça, nem mesmo nível sócio-econômico. As causas podem ser aparentes como doenças, traumas, cirurgias, pode ser de origem psicológica e emocional ou ainda ser a própria doença sem causa relacionada. O conceito de dor da Associação Internacional para o Estudo da Dor (IASP) de 1986, que assumiremos neste estudo, refere-se à dor como uma experiência sensorial e emocional desagradável que é descrita em termos de lesões teciduais, reais ou potenciais. A dor é sempre subjetiva e cada indivíduo aprende e utiliza este termo a partir de suas experiências traumáticas (MERSKEY, 1994). Nesse conceito, a dor é compreendida como um fenômeno multifatorial, e a lesão tecidual, aspectos emocionais, socioculturais e ambientais são fatores que compõem o fenômeno (VON KORFF, 1990; PIMENTA, 1994).

Atualmente, a dor está sendo considerada como o quinto sinal vital, assim como freqüência cardíaca, pressão arterial, frequiência respiratória e temperatura.

Quando a dor está presente, causa sofrimento, desgaste físico, psíquico e social, piora a qualidade de vida, interfere na produtividade no emprego e muitas vezes acarreta licenças médicas e aposentadorias por doenças (PIMENTA, 1994).

Quando não controlada, a dor causa o aumento de complicações pós-operatórias prolongando as internações, onerando ainda mais o sistema de saúde, além de ser causa de insatisfação do doente com a assistência recebida (PIMENTA, 2001).
Estimativas da OMS (Organização Mundial da Saúde) prevêem 10 milhões de novos casos de câncer diagnosticados no mundo a cada ano; em 1/3 desses casos, o alívio do sofrimento poderia ser alcançado por meio do controle da dor e outros sintomas (SCHAELLER, 2002).

Estudos epidemiológicos sobre dor no Brasil são raros. Em pesquisa realizada na cidade de Londrina Pr, envolvendo populações não vinculadas a serviços de saúde, em todas as faixas etárias, foram encontrados resultados que demonstram a magnitude do problema. Foram avaliadas 915 crianças escolares entre 7 e 14 anos e observou-se uma prevalência de dor recorrente de $28,75 \%$ (ROSSETO, 2001). Entre os 505 adultos funcionários de uma Universidade, observou-se dor crônica em $61,38 \%$. Entre os adultos, a dor de cabeça foi a mais prevalente $(26,36 \%)$, seguida de dor lombar $(19,4 \%)$ e em membros inferiores $(13,26 \%)$ (KRELING, 2001). Dos 451 idosos entrevistados, $51,44 \%$ tinham dor crônica, os locais mais prevalentes foram região dorsal $(21,73 \%)$, membros inferiores $(21,50 \%)$ e região cefálica $(7,09 \%)$ (DELLAROZA, 2001). Nas três populações estudadas, foi possível comprovar o prejuízo advindo da dor para as atividades escolares, laborativas, sono, humor e lazer.

Apesar da alta prevalência e do comprometimento as funcionalidades físicas, psíquicas e sociais decorrentes da dor e de outros sintomas incapacitantes, o tema é, geralmente, pouco enfatizado nos cursos de graduação na área de Saúde (PIMENTA, 2001). Tal fato tem conseqüências diretas na assistência prestada ao indivíduo que muitas vezes tem sua dor subtratada. Segundo Teixeira (1997), maior contingente das síndromes álgicas pode ser controlado com medidas farmacológicas e físicas simples que estão ao alcance de todos os profissionais de saúde.

A Universidade, como formadora de profissionais de saúde, tem a responsabilidade de formar estes com conceitos básicos sobre dor e terapêuticas para o controle. Profissionais mais capacitados no controle da dor certamente garantirão uma atenção mais humanizada a população. 
A Universidade Estadual de Londrina (UEL), forma anualmente grande quantidade de profissionais para o mercado de trabalho. $\mathrm{Na}$ área da Saúde, por exemplo, são 400 alunos por ano. Estes profissionais vão estar atuando nos serviços de saúde de todo o país, e esse fato reforça a importância de se conhecer o tipo de embasamento científico e prático proporcionado pela graduação para assuntos pertinentes ao controle da dor.

O preparo adequado dos profissionais de saúde, com embasamento científico e atuação prática poderiam melhorar a assistência e o controle da dor. No entanto, alguns profissionais, por estarem despreparados para lidar com as queixas álgicas, podem estar subtratando a dor dos pacientes, especialmente, em se tratando de dor crônica. A falta de informação é apontada como a principal, entre as múltiplas causas do inadequado alívio da dor.

Sabe-se que conceitos referentes à dor podem ser tratados em diferentes disciplinas e atividades nos curso de graduação, e que, o conjunto desta aprendizagem formará a base de conceitos e competências que esse aluno aplicará em sua vida profissional.

Apesar do tema dor constar em ementas e conteúdos programáticos dos diversos cursos da área de saúde, pouco se sabe como estes temas são abordados.

Assim faz-se essencial avaliar o que tem sido aprendido por estes profissionais durante sua formação. Um diagnóstico adequado dos conceitos adquiridos pelos alunos da área de saúde durante a graduação pode ser a base para que se realizem adequações necessárias nos currículos e disciplinas, visando à formação de profissionais mais preparados para realizar o controle da dor.

Os objetivos deste estudo foram: identificar as disciplinas e conteúdos sobre o tema dor, identificar os conceitos adquiridos pelos alunos referentes aos aspectos gerais da dor, à avaliação da dor e às terapêuticas analgésicas.

\section{Método}

O presente estudo caracterizou-se como transversal e descritivo.

\section{Local e População}

A pesquisa foi realizada na UEL, com alunos do último ano da área da Saúde, dos cursos de Enfermagem, Farmácia, Fisioterapia, Medicina, Psicologia e Odontologia, totalizando 189 estudantes entrevistados.Para realização dos trabalhos, houve a aprovação pelo Comitê de Ética em pesquisa da Universidade Estadual de Londrina.

\section{Coleta de Dados}

A coleta de dados foi realizada durante o segundo semestre de 2002, por meio de um questionário com questões objetivas.

\section{Instrumento}

Para a coleta de dados, foi utilizado um instrumento anteriormente testado mediante a aplicação tanto a profissionais de saúde, formado por quatro partes (Anexo 01). Os alunos foram esclarecidos quanto aos objetivos da pesquisa e participaram somente participaram após assinarem o termo de consentimento (Anexo 02).

A primeira parte do questionário visou a obter dados de identificação pessoal como idade, sexo e curso de graduação.

Foram pesquisados os temas sobre dor abordados na graduação, e o conhecimento sobre aspectos gerais da dor e terapêuticas farmacológicas e não farmacológicas.

\section{Análise dos Dados}

O banco de dados foi organizado em planilhas do Programa Excel - Windows 98, o que possibilitou organização dos dados em gráficos e tabelas. 
Os itens do questionário foram agrupados em aspectos relacionados a: fisiologia da dor, avaliação e subjetividade da dor, terapêuticas farmacológicas e não-farmacológicas. Esses itens foram estabelecidos com o objetivo de facilitar a análise dos resultados.

A análise estatística foi realizada por meio de frequiência absoluta e relativa, além de medidas de dispersão, quando pertinentes.

\section{Resultados e Discussão}

A pesquisa foi realizada durante os meses de dezembro de 2002 e março de 2003. Dos 380 estudantes, $49,7 \%$ responderam ao questionário. A perda de $51 \%$ explica-se pela dificuldade de acesso aos alunos no último ano dos cursos já que estes realizam estágios em diferentes campos e locais.

A população constitui-se de $63 \%$ de estudantes do sexo feminino e $37 \%$ do sexo masculino, com idades variando de 20 a 44 anos, sendo a média de idade 23,18 e desvio padrão de 2,04.

Nos gráficos 1 e 2, são apresentados a distribuição dos participantes por curso e os temas referentes a dor abordados durante a graduação.

Gráfico 1. Distribuição dos participantes por curso Londrina 2003

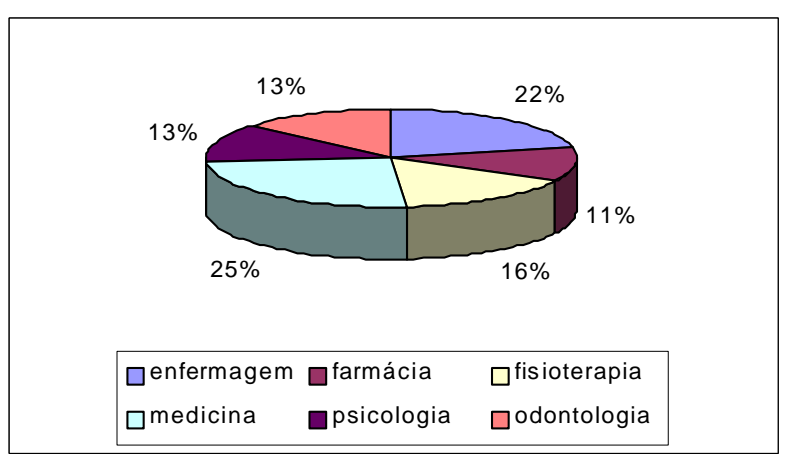

Gráfico 2. Distribuição dos temas sobre dor abordados durante a graduação-Londrina 2003.

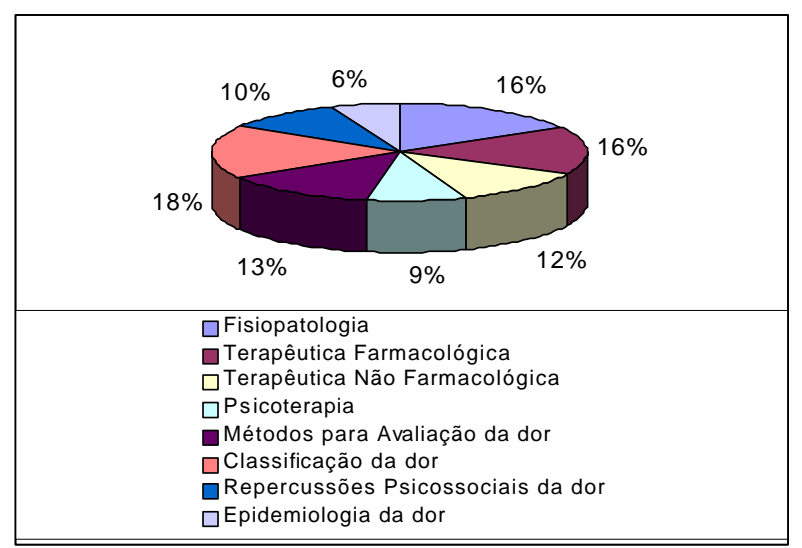

A explicação dos dados apresentados no gráfico 02 recai sobre o fato de os conteúdos terem sido abordados como temas principais, mas como conceitos complementares em diversas disciplinas. Tal forma de abordagem dificultaria o reconhecimento pelos alunos dos temas abordados na graduação.

A grande maioria dos acadêmicos $(87,8 \%)$, não participou de eventos e cursos sobre dor. Entre os estudantes que participaram, o curso de Enfermagem obteve a maior porcentagem com $26,8 \%$, seguido do curso de Odontologia com $16 \%$. A Medicina teve como resultado apenas $8,5 \%$ e os estudantes de Fisioterapia não relataram nenhuma participação em qualquer evento relacionado à dor.

Quanto a opinião se a dor poderia ser considerada como um sinal vital, tal qual pressão arterial, freqüência respiratória, freqüência cardíaca e temperatura, $53,4 \%$ dos participantes está de acordo, porém $33,3 \%$ não concordam com a afirmativa e $13,2 \%$ não souberam responder. Conforme indicação da Agência Americana de Pesquisa e Qualidade em Saúde Pública, a Sociedade Americana de Dor e da Sociedade Brasileira de Estudo da Dor, é essencial que os serviços e profissionais passem a considerar a dor como o $5^{\circ}$ sinal vital, visando à melhoria $\mathrm{e}$ humanização da assistência (BRAUN, 2000; ANDRADE, 2000). 
Tabela 1. Distribuição das respostas relacionadas a fisiopatologia da dor-Londrina 2003

\begin{tabular}{lcccc}
\hline \multicolumn{1}{c}{$\begin{array}{c}\text { Aspectos relacionados a } \\
\text { Fisiopatologia da dor }\end{array}$} & Concorda & $\begin{array}{c}\text { Concorda } \\
\text { Parcialmente }\end{array}$ & Discorda & $\begin{array}{c}\text { Não } \\
\text { Sabe }\end{array}$ \\
\hline $\begin{array}{l}\text { A intensidade da dor está } \\
\text { diretamente relacionada com } \\
\text { o grau de lesão do tecido. }\end{array}$ & $18 \%$ & $38,6 \%$ & $40 \%{ }^{* *}$ & $3,4 \%$ \\
\hline $\begin{array}{l}\text { O doente que depois de tomar } \\
\text { um placebo afirma que a dor } \\
\text { melhorou, não tinha } \\
\text { realmente dor. }\end{array}$ & $6,3 \%$ & $18 \%$ & $73,5 \% * *$ & $2,1 \%$ \\
\hline $\begin{array}{l}\text { A origem da dor pode ser } \\
\text { psicológica ou emocional. }\end{array}$ & $87,3 \%^{* *}$ & $11,1 \%$ & $1,05 \%$ & $0,5 \%$ \\
\hline $\begin{array}{l}\text { Existem casos em que a dor é } \\
\text { a própria doença e não um } \\
\text { sintoma secundário. }\end{array}$ & $56,6 \% * *$ & $15,8 \%$ & $18 \%$ & $9,5 \%$ \\
\hline $\begin{array}{l}\text { A dor pode ocorrer em um } \\
\text { local mesmo sem lesão } \\
\text { aparente. }\end{array}$ & $95,7 \% * *$ & $2,6 \%$ & $0 \%$ & $1,6 \%$ \\
\hline
\end{tabular}

Na Tabela 01, pode-se observar que na questão sobre a relação entre a intensidade da dor e o grau de lesão do tecido $38,6 \%$ dos estudantes tiveram dúvida na resposta, $40 \%$ discordaram dessa relação e $18 \%$ concordaram com ela. Segundo Teixeira (1997), dor é uma qualidade sensorial complexa, freqüentemente não relacionada ao grau de lesão tecidual. Porém, pelo que foi observado nas respostas, este não é um conceito aprendido por todos, já que os valores dos que concordam parcialmente e dos que concordam com esta afirmação somaram somente $57 \%$ dos estudantes.

Os valores assinalados com asteriscos (**), salientam as respostas corretas, o que demonstra um bom nível de conhecimento dos participantes, já que a maioria respondeu de maneira adequada as afirmações. Tal achado é muito importante pois o adequado conhecimento sobre fisiopatologia da dor é a base para uma assistência de melhor qualidade

De acordo com Teixeira (1999), a interpretação dolorosa envolve aspectos sensitivos, cognitivos, comportamentais e culturais. É influenciada por fatores sócio-econômicos, ambiente familiar, estratégias de enfrentamento e compensações. Concordaram que a dor é influenciada pela cultura $79,4 \%$ dos estudantes, o que demonstra adequada compreensão da multidimensionalidade da dor.

O indivíduo tem toda uma história de vida e uma maneira própria e única de sentir a dor e isso pode
Tabela 2. Distribuição das respostas relacionadas a subjetividade e avaliação da dor - Londrina 2003

\begin{tabular}{lcccc}
\hline $\begin{array}{l}\text { Aspectos relacionados } \\
\text { a subjetividade e } \\
\text { avaliação da dor }\end{array}$ & Concorda & $\begin{array}{c}\text { Concorda } \\
\text { Parcialmente }\end{array}$ & Discorda & $\begin{array}{c}\text { Não } \\
\text { Sabe }\end{array}$ \\
\hline $\begin{array}{l}\text { A dor deve ser avaliada } \\
\text { sistematicamente. }\end{array}$ & $75,6 \% * *$ & $14,8 \%$ & $2,7 \%$ & $6,8 \%$ \\
\hline $\begin{array}{l}\text { A cultura influencia na } \\
\text { expressão da dor. }\end{array}$ & $79,4 \% * *$ & $14,2 \%$ & $4,2 \%$ & $2,1 \%$ \\
\hline $\begin{array}{l}\text { O paciente é a maior } \\
\text { autoridade para } \\
\text { informar sobre sua dor. }\end{array}$ & $75,1 \% * *$ & $20,1 \%$ & $4,8 \%$ & $0 \%$ \\
\hline $\begin{array}{l}\text { Embora o limiar de } \\
\text { percepção à dor seja } \\
\text { semelhante entre os } \\
\text { indivíduos, a tolerância }\end{array}$ & $88,3 \% * *$ & $5,8 \%$ & $5,8 \%$ & $0 \%$ \\
à dor varia muito. & & & & \\
\hline
\end{tabular}

ter um significado diferente para cada pessoa. Os estudantes concordam com este princípio, quando observa-se que $75,1 \%$ dos estudantes reconheceram o paciente como autoridade sobre sua dor, e que $88,3 \%$ reconhecem que a tolerância a dor varia para cada indivíduo (TEIXEIRA, 1999).

Tabela 3. Distribuição da respostas relacionadas a terapêutica da dor - Londrina 2003

\begin{tabular}{lllll}
\hline $\begin{array}{l}\text { Aspectos relacionados } \\
\text { a terapêutica da dor }\end{array}$ & Concorda & $\begin{array}{c}\text { Concorda } \\
\text { Parcialmente }\end{array}$ & Discorda & $\begin{array}{c}\text { Não } \\
\text { Sabe }\end{array}$ \\
\hline $\begin{array}{l}\text { É preciso curar a } \\
\text { enfermidade e não a } \\
\text { dor. }\end{array}$ & $30,1 \%$ & $27 \%$ & $39,1 \%$ & $3,7 \%$ \\
\hline $\begin{array}{l}\text { De uma maneira geral, } \\
\text { o paciente com dor é }\end{array}$ & $7,9 \%$ & $39,1 \%$ & $50,8 \%$ & $2,1 \%$ \\
assistido com eficácia. & & & & \\
\hline $\begin{array}{l}\text { A dor pode ser tratada } \\
\text { mesmo antes de se } \\
\text { conhecer a sua causa. }\end{array}$ & $55,5 \%$ & $32,8 \%$ & $9,5 \%$ & $2,1 \%$ \\
\hline
\end{tabular}

Diferentemente do observado quanto a fisiologia, subjetividade e avaliação da dor, no qual os alunos demonstraram conhecimentos adequados, as respostas referentes a terapêutica demonstraram fragilidade de conceitos.

No primeiro aspecto avaliado (necessidade de curar a enfermidade e não a dor), chama a atenção: $30,1 \%$ dos estudantes responderem que é preciso curar a doença, mas não a dor. É evidente que a preocupação com a cura da enfermidade deva existir, mas esta ação não deve ser separada do controle da dor. Muitas vezes é essencial controlar a dor, já que muitas enfermidades que provocam dores crônicas não podem se curadas. 
Observa-se que $32,8 \%$ concordam parcialmente e 9,5\% discordam que a dor possa ser trata mesmo antes de se conhecer a causa. Sabe-se que o controle da dor deve ser uma prioridade mesmo em situações na qual não se conhece a causa. Exemplo disso são as situações de emergência, nas quais um paciente com analgesia adequada pode facilitar as intervenções e ter menor risco de complicações (MELZACK, 1982). Além disso, nem sempre é possível determinar com precisão a causa, mas é essencial controlar a dor, para garantir qualidade de vida.

Observa-se que a maioria dos estudantes $(50,8 \%)$, discorda que o paciente com dor é assistido de maneira eficaz. Espera-se que a consciência frente a esta situação, aqui demonstrada, possa transformar estes futuros profissionais em agentes de mudança, para que a qualidade de assistência possa ser melhorada.

Tabela 4. Distribuição das respostas relacionadas a terapêuticas farmacológicas no controle dor - Londrina 2003.

\begin{tabular}{llccc}
\hline $\begin{array}{l}\text { Aspectos relacionadas a } \\
\text { terapêuticas farmacológicas } \\
\text { para o controle da dor }\end{array}$ & Concorda & $\begin{array}{c}\text { Concorda } \\
\text { Parcialmente }\end{array}$ & Discorda & $\begin{array}{c}\text { Não } \\
\text { Sabe }\end{array}$ \\
\hline $\begin{array}{l}\text { Se a dor for suportável, é } \\
\text { melhor não medicar e } \\
\text { investigar a causa. }\end{array}$ & $30,7 \%$ & $31,7 \%$ & $33,3 \%$ & $4,2 \%$ \\
\hline $\begin{array}{l}\text { É melhor utilizar analgésicos } \\
\text { em horários fixos para }\end{array}$ & $21,6 \%$ & $32,8 \%$ & $33,3 \%$ & $12,1 \%$ \\
pacientes com risco de dor. & & & & \\
\hline $\begin{array}{l}\text { Orientar o doente a tolerar a } \\
\text { dor e espaçar o uso de } \\
\text { analgésicos é uma forma de } \\
\text { evitar medicação em excesso. }\end{array}$ & $36,5 \%$ & $36 \%$ & $16,4 \%$ & $11,1 \%$ \\
\hline $\begin{array}{l}\text { Os analgésicos podem viciar. } \\
\text { Quando a prescrição do }\end{array}$ & $39,7 \%$ & $23,2 \%$ & $22,7 \%$ & $14,2 \%$ \\
$\begin{array}{l}\text { analgésico é em esquema "se } \\
\text { necessário", devemos sempre } \\
\text { esperar a solicitação do } \\
\text { paciente. }\end{array}$ & $38 \%$ & $31,2 \%$ & $22,7 \%$ & $7,9 \%$ \\
\hline $\begin{array}{l}\text { Os analgésicos anti- } \\
\text { inflamatórios possuem dose } \\
\text { máxima, ou seja, a partir de } \\
\text { determinada dosagem não } \\
\text { produzem maior efeito } \\
\text { analgésico. }\end{array}$ & $49,7 \%$ & $14,2 \%$ & $7,4 \%$ & $28,5 \%$ \\
\hline
\end{tabular}

O conhecimento sobre terapêutica ainda demonstra fragilidades e, talvez, devesse ser mais enfatizado. Observa-se, na Tabela 04, que muitos estudantes ainda possuem conceitos errôneos sobre o uso de analgésicos, $33,3 \%$ consideram que é melhor suportar a dor até a confirmação de sua causa, apenas $21,6 \%$ concordam que é melhor prevenir a dor com uso de medicação em horário fixo. Em relação ao fato de que o doente deva tolerar a dor para evitar o uso excessivo de analgésicos, a porcentagem que concorda $36,5 \%$ ficou semelhante a dos que tem dúvidas $36 \%$, o que demonstra uma inadequação de conhecimentos sobre a importância do total controle da dor. É necessário salientar todos os transtornos gerados pela sensação dolorosa, (estresse, queda da produtividade, alteração do humor, entre outras), fato que justificaria o início da medicação assim que possível, visando à sua prevenção. Nenhum indivíduo precisa passar por esta "dor suportável”, o correto é o alívio desta sensação, mas existe toda uma questão cultural na qual as pessoas acreditam que alguns tipos de dor são inevitáveis, principalmente após atos cirúrgicos, dores crônicas em idosos e dores relacionadas ao câncer.

Vale salientar que o ideal é a utilização de esquemas de horário de analgésico, mas quando prescrito "se necessário" deve-se aguardar a solicitação, como foi reconhecido por $38 \%$ dos acadêmicos.

Os acadêmicos demonstraram adequados conhecimentos a ceca da farmacologia dos analgésicos: $49,7 \%$ concordaram que os antiinflamatórios possuem dose teto.

Tabela 5. Distribuição das respostas relacionadas a terapêuticas não farmacológicas para o controle da dor Londrina 2003.

\begin{tabular}{lcccc}
\hline $\begin{array}{l}\text { Aspectos relacionadas a } \\
\text { terapêuticas não Farmacológica } \\
\text { para o controle da dor }\end{array}$ & Concorda & $\begin{array}{c}\text { Concorda } \\
\text { Parcialmente }\end{array}$ & Discorda & $\begin{array}{c}\text { Não } \\
\text { Sabe }\end{array}$ \\
\hline $\begin{array}{l}\text { A acupuntura é uma indicação útil } \\
\text { para alguns casos de dor. }\end{array}$ & $69,8 \%$ & $7,9 \%$ & $4,2 \%$ & $18 \%$ \\
\hline $\begin{array}{l}\text { A psicoterapia é indicada somente } \\
\text { em casos onde não se descobrem } \\
\text { causas biológicas. }\end{array}$ & $14,8 \%$ & $21,6 \%$ & $51,8 \%$ & $11,6 \%$ \\
\hline $\begin{array}{l}\text { Técnicas como aplicação de calor, } \\
\text { frio e massagem são alternativas } \\
\text { terapêuticas para dor. }\end{array}$ & $36 \%$ & $12,7 \%$ & $45 \%$ & $6,3 \%$ \\
\hline $\begin{array}{l}\text { Técnicas de relaxamento só são } \\
\text { indicadas para o alívio de tensão } \\
\text { emocional. }\end{array}$ & $12,1 \%$ & $24,3 \%$ & $60,3 \%$ & $4,2 \%$ \\
$\begin{array}{l}\text { As técnicas se distração podem } \\
\text { promover o alívio da dor. }\end{array}$ & $53 \%$ & $32,8 \%$ & $4,2 \%$ & $10 \%$ \\
\hline
\end{tabular}


Grande parte dos participantes concordou com o uso de terapêuticas não farmacológicas para o controle da dor. $\mathrm{O}$ uso de acupuntura e técnicas de distração foram as que obtiveram maior aceitação, com $69,8 \%$ e $53 \%$, respectivamente. A maioria dos acadêmicos concordou que técnicas de relaxamento e psicoterapia têm múltiplas indicações. Menor concordância ocorreu frente as terapêuticas de aplicação de calor e frio, já que $36 \%$ concordaram e $45 \%$ discordaram que estas possam ser terapêuticas analgésicas coadjuvantes. Tais técnicas de estimulação cutânea podem proporcionar alívio mais prolongado da dor . As compressas frias, por exemplo, atuam diminuindo a velocidade de condução dos estímulos dolorosos (PIMENTA, 1997).

De acordo com Pimenta (1997), a liberação de estresse e tensão através de técnicas de relaxamento podem proporcionar alívio da dor. O relaxamento pode diminuir a ansiedade, o tono muscular, a fadiga e, conseqüentemente, aliviar a dor.

Tabela 6. Distribuição das respostas relacionadas ao uso de opióides no controle da dor - Londrina 2003

\begin{tabular}{lcccc}
\hline $\begin{array}{l}\text { Aspectos relacionadas ao } \\
\text { uso de opiódes para o } \\
\text { controle da dor }\end{array}$ & Concorda & $\begin{array}{c}\text { Concorda } \\
\text { Parcialmente }\end{array}$ & Discorda & $\begin{array}{c}\text { Não } \\
\text { Sabe }\end{array}$ \\
\hline $\begin{array}{l}\text { Opióides fortes como a } \\
\text { meperidina podem causar } \\
\text { tolerância, mas não } \\
\text { dependência física. }\end{array}$ & $17 \%$ & $11,6 \%$ & $43,4 \%$ & $28 \%$ \\
\hline $\begin{array}{l}\text { A dependência psíquica é } \\
\text { rara com o uso de morfina. }\end{array}$ & $8,5 \%$ & $11,1 \%$ & $62 \%$ & $18,5 \%$ \\
\hline $\begin{array}{l}\text { A morfina deve ser } \\
\text { utilizada somente como }\end{array}$ & $36 \%$ & $25 \%$ & $20 \%$ & $19 \%$ \\
última alternativa. & & & & \\
\hline $\begin{array}{l}\text { A morfina apresenta alto } \\
\text { risco de depressão } \\
\text { respiratória. }\end{array}$ & $49,2 \%$ & $13,2 \%$ & $4,8 \%$ & $32,8 \%$ \\
\hline
\end{tabular}

Quanto aos conceitos referentes a utilização de opióides, o panorama apresentado é bastante preocupante. A maior parte dos alunos discorda que os opióides possam causar tolerância $(43,4 \%)$, discordam que a dependência psíquica é rara (62\%) e concordaram que há alto risco de depressão respiratória $(49,2 \%)$. Além disso, $36 \%$ acham que a morfina só pode ser usada em última alternativa e $25 \%$ concordam parcialmente com esta afirmação.
Os conceitos apresentados pelos alunos confirmam as discussões que falsos "tabus" perante a utilização de opióides são os principais obstáculos para uma adequada analgesia, no Brasil, dos clientes com dores malignas e intensas.

No $1^{\circ}$ Consenso Nacional de Dor Oncológica, Schaeller (2002) apresenta as barreiras para o manejo da dor oncológica e destaca o inadequado conhecimento sobre farmacologia e efeitos colaterais dos opióides como barreira tanto para médicos como enfermeiros.

A farmacodinâmica e a experiência clínica com o uso de opióides confirmam que tanto a tolerância como a dependência física podem ocorrer, mas não devem impedir sua utilização terapêutica, além disso, a dependência psíquica é rara (GIUBLIN, 2002).

\section{Conclusão}

Com o presente estudo, verifica-se que o tema dor é abordado nos cursos de graduação da área da saúde, porém, não como um tema principal, mas como um subtema em diversos conteúdos. Os alunos demonstraram adequados conhecimentos na fisiologia e avaliação da dor, contudo demonstraram fragilidades em conceitos referentes a terapêuticas. É extremamente relevante a importância que o tema dor representa para estes acadêmicos, já que estarão cuidando da população acometida. Assim, faz-se essencial rever como o tema é abordado nos cursos de graduação em saúde. Limitações deste estudo foram a baixa porcentagem de alunos que responderam ao questionário e a não avaliação, através dos docentes e programas de disciplinas, quanto a forma de abordagem do tema. Novas pesquisas poderão ser realizadas, visando a um maior aprofundamento das questões aqui elaboradas. 


\section{Referências}

ANDRADE, M. P. O novo milênio e a dor como o quinto sinal vital. Dor, Curitiba, v.2, p.1-3, 2000.

BRAUN, L. Tratamento da dor ainda é desconhecido no país. Dor em foco, Curitiba, v.3, n.2, p.1-2, 2000.

DELLAROZA, M. S. G. Prevalência e caracterização da dor crônica em idosos servidores municipais da Londrina. 2001. Dissertação (Mestrado) - Escola de Enfermagem da Universidade de São Paulo, São Paulo, 2001.

GIUBLIN, M. L. Tratamento farmacológico. In: CONSENSO NACIONAL DE DOR ONCOLÓGICA, 1., 2000, São Paulo. Anais... São Paulo: SBDE, 2002. p.13-18.

KRELING, M. C. G. D. Prevalência de dor crônica em adultos trabalhadores. 2000. Dissertação (Mestrado) Escola de Enfermagem, Universidade de São Paulo, São Paulo.

MELZACK, R.; WALL, P. D.; TY, T. C. Acute pain in an emergency clinic: latency of onset and descriptor patterns related to different injuries. Pain, Amsterdam, n.14, p.3343, 1982.

MERSKEY, N. B. Classification of chronic pain : descriptions of chronic pain syndromes and definitions of pain terms prepared by the Internacional Association for the Study of Pain. 2. ed. Seattle: IASP Press; 1994.
PIMENTA, C. A. M.; TEIXEIRA, M. J. Avaliação da dor. Revista Médica, São Paulo, v.76, n.1, p.27-35, jan./fev 1997.

PIMENTA,C. A. M. Dor: manual clínico de enfermagem. São Paulo: [s. n.], 2000.

PIMENTA, C. A. M. Escalas de avaliação de dor. In: TEIXEIRA, M. J.; CORREAA, C. F.; PIMENTA,C. A. M. Dor: conceitos gerais. São Paulo: Limay, 1994. cap.4, p.7186.

PIMENTA, C. A. M. Proposta de conteúdo mínimo sobre dor e cuidados paliativos nos cursos de graduação da área de saúde. Revista Medica, São Paulo, v.2, n.1, p.2335, 2001.

ROSSETTO, E. G. Dor recorrente em escolares na cidade de Londrina: prevalência, caracterização e impacto nas atividades diárias. 2001. Dissertação (Mestrado) - Escola de Enfermagem, Universidade de São Paulo, São Paulo.

SCHAELLER, M. T. Dor oncológica. In: CONSENSO NACIONAL DE DOR ONCOLÓGICA, 1., 2002, São Paulo. Anais... São Paulo: SBDE, 2002. p.51-66.

TEIXEIRA, M. J. Fisiopatologia da dor. Revista Medica, São Paulo, v.76, n.7, p.20, 1997.

TEIXEIRA, M. J. Avaliação da dor: fundamentos teóricos e análise crítica. Revista Medica, São Paulo, v.78, n.2, p.32$189,1999$.

VON KORFF, M.; DWOKIN, S. F.; LE RESCHE, L. Graded chronic pain status an epidemiologic evaluation. Pain, Amsterdam, v.40, p.279-291, 1990. 


\section{ANEXO 01}

\section{Instrumento de coleta de dados}

1-Sexo ( ) Feminino ( ) Masculino

2-Idade

3-Curso
( ) Enfermagem
( ) Medicina
( ) Farmácia
( ) Psicologia
( ) Fisioterapia
( ) Odontologia

4-O tema "dor" foi abordado de maneira específica em sua formação regular?

( ) $\operatorname{Sim}$ ( ) Não

Se sim, quais aspectos foram abordados?

( ) Fisiopatologia

( ) Terapêutica Farmacológica

( ) Terapêutica Não Farmacológica

( ) Psicoterapia

( ) Métodos para avaliação da dor

( ) Classificação da dor ( aguda, crônica, recorrente...)

( ) Repercussões Psicossociais da dor

( ) Epidemiologia da dor

5- Já participou de algum curso sobre dor?

( ) $\operatorname{Sim}($ ) Não

Se sim, promovido por quem?

6- Você acha que a dor pode ser considerada como um sinal vital, tal qual pressão arterial, freqüência respiratória, frequiência cardíaca, temperatura?

( ) $\operatorname{Sim}$ ( ) Não ( ) Não sabe 
7- De acordo com seus conhecimentos, emita sua opinião em relação às afirmativas abaixo sobre os aspectos gerais da dor:

\begin{tabular}{l|l|l|l|l|}
\hline \multicolumn{1}{|c|}{ Aspectos Gerais } & Concorda & $\begin{array}{c}\text { Concorda } \\
\text { Parcialmente }\end{array}$ & Discorda & $\begin{array}{c}\text { Não } \\
\text { Sabe }\end{array}$ \\
\hline $\begin{array}{l}\text { A intensidade da dor está diretamente } \\
\text { relacionada com o grau de lesão do tecido. }\end{array}$ & & & \\
\hline $\begin{array}{l}\text { O doente que depois de tomar um placebo } \\
\text { afirma que a dor melhorou, não tinha realmente } \\
\text { dor. }\end{array}$ & & & & \\
\hline $\begin{array}{l}\text { A origem da dor pode ser psicológica ou } \\
\text { emocional. }\end{array}$ & & & & \\
\hline $\begin{array}{l}\text { Se a dor for suportável, é melhor não medicar e } \\
\text { investigar a causa. }\end{array}$ & & & & \\
\hline $\begin{array}{l}\text { É preciso curar a enfermidade e não a dor. } \\
\text { Existem casos em que a dor é a própria doença } \\
\text { e não um sintoma secundário. }\end{array}$ & & & & \\
\hline $\begin{array}{l}\text { A dor pode ocorrer em um local mesmo sem } \\
\text { lesão aparente. }\end{array}$ & & & & \\
\hline A dor deve ser avaliada sistematicamente. & & & & \\
\hline $\begin{array}{l}\text { A cultura influencia na expressão da dor. } \\
\text { O paciente é a maior autoridade para informar } \\
\text { sobre sua dor. }\end{array}$ & & & & \\
\hline $\begin{array}{l}\text { Embora o limiar de percepção à dor seja } \\
\text { semelhante entre os indivíduos, a tolerância à } \\
\text { dor varia muito. }\end{array}$ & & & & \\
\hline $\begin{array}{l}\text { De uma maneira geral, o paciente com dor é } \\
\text { assistido com eficácia. }\end{array}$ & & & & \\
\hline
\end{tabular}


8-Complete o quadro abaixo quanto às terapêuticas de dor.

\begin{tabular}{|c|c|c|c|c|}
\hline Terapêuticas de dor & Concorda & $\begin{array}{c}\text { Concorda } \\
\text { Parcialmente }\end{array}$ & Discorda & $\begin{array}{l}\text { Não } \\
\text { Sabe }\end{array}$ \\
\hline \multicolumn{5}{|l|}{$\begin{array}{l}\text { A dor pode ser tratada mesmo antes de } \\
\text { se conhecer a sua causa. }\end{array}$} \\
\hline \multicolumn{5}{|l|}{$\begin{array}{l}\text { É melhor utilizar analgésicos em } \\
\text { horários fixos para pacientes com risco } \\
\text { de dor. }\end{array}$} \\
\hline \multicolumn{5}{|l|}{$\begin{array}{l}\text { Orientar o doente a tolerar a dor e } \\
\text { espaçar o uso de analgésicos é uma } \\
\text { forma de evitar medicação em excesso. }\end{array}$} \\
\hline \multicolumn{5}{|l|}{ Os analgésicos podem viciar. } \\
\hline \multicolumn{5}{|l|}{$\begin{array}{l}\text { Opióides fortes como a meperidina } \\
\text { podem causar tolerância, mas não } \\
\text { dependência física. }\end{array}$} \\
\hline \multicolumn{5}{|l|}{$\begin{array}{l}\text { Quando a prescrição do analgésico é } \\
\text { em esquema "se necessário", devemos } \\
\text { sempre esperar a solicitação do } \\
\text { paciente. }\end{array}$} \\
\hline \multicolumn{5}{|l|}{$\begin{array}{l}\text { A acupuntura é uma indicação útil para } \\
\text { alguns casos de dor. }\end{array}$} \\
\hline \multicolumn{5}{|l|}{$\begin{array}{l}\text { A dependência psíquica é rara com o } \\
\text { uso de morfina. }\end{array}$} \\
\hline \multicolumn{5}{|l|}{$\begin{array}{l}\text { A morfina deve ser utilizada somente } \\
\text { como última alternativa. }\end{array}$} \\
\hline \multicolumn{5}{|l|}{$\begin{array}{l}\text { A morfina apresenta alto risco de } \\
\text { depressão respiratória. }\end{array}$} \\
\hline \multicolumn{5}{|l|}{$\begin{array}{l}\text { Os analgésicos anti-inflamatórios } \\
\text { possuem dose máxima, ou seja, a partir } \\
\text { de determinada dosagem não } \\
\text { produzem maior efeito analgésico. }\end{array}$} \\
\hline \multicolumn{5}{|l|}{$\begin{array}{l}\text { A psicoterapia é indicada somente em } \\
\text { casos onde não se descobrem causas } \\
\text { biológicas. }\end{array}$} \\
\hline \multicolumn{5}{|l|}{$\begin{array}{l}\text { Técnicas como aplicação de calor, frio } \\
\text { e massagem são alternativas } \\
\text { terapêuticas para dor. }\end{array}$} \\
\hline \multicolumn{5}{|l|}{$\begin{array}{l}\text { Técnicas de relaxamento só são } \\
\text { indicadas para o alívio de tensão } \\
\text { emocional. }\end{array}$} \\
\hline $\begin{array}{l}\text { As técnicas se distração podem } \\
\text { promover o alívio da dor. }\end{array}$ & & & & \\
\hline
\end{tabular}




\section{ANEXO 02 \\ Termo de Consentimento}

O projeto de Pesquisa: "O que está sendo aprendido sobre dor na UEL?" realizado pela aluna de Enfermagem Talita Woitas Sereza, sobre a coordenação da professora Mara Solange Gomes Dellaroza do Departamento de Enfermagem, tem como objetivo avaliar o que está sendo aprendido sobre dor nos cursos da área de Saúde da Universidade Estadual de Londrina.

O método de coleta de dados é através de questionário a ser respondido pelos alunos do último ano dos cursos de saúde.

Assim solicito sua colaboração no sentido de responder ao questionário em anexo.

Como pesquisadora responsável asseguro que:

1- O informante não será identificado.

2- A utilização das informações será exclusivamente para fins de pesquisa.

3- A participação ou não nesta pesquisa não implicará em qualquer prejuízo.

Mara S. G. Dellaroza; Talita W. Sereza

Ciente das informações acima, eu $\mathrm{RG}$

concordo em participar da pesquisa "O que tem sido aprendido sobre dor na UEL."

Londrina, de de 